\title{
Blood-brain barrier disruption caused by neonatal sevoflurane- induced depends on exposure time and is reversible in mice
}

\author{
Zhongliang Sun ${ }^{1, *}$, Maiko Satomoto ${ }^{2, *}$, Yushi U Adachi ${ }^{3}$, and Koshi Makita ${ }^{4, *}$ \\ Department of Anesthesiology, ${ }^{1}$ The First Affiliated Hospital of Dalian Medical University, Dalian, China, ${ }^{2}$ Toho \\ University School of Medicine, Tokyo, ${ }^{3}$ Graduate School of Medicine, Nagoya University, Aichi, ${ }^{4}$ Sasaki Foundation \\ Kyoundo Hospital, Tokyo, Japan
}

Neonatal exposure to anesthetics, such as sevoflurane, induces neuronal apoptosis and results in learning deficits in rodents $[1,2]$. We showed that exposure to $2 \%$ sevoflurane for $6 \mathrm{~h}$ resulted in blood-brain barrier (BBB) disruption in the hippocampus of postnatal day 6 (POD6) mice [3]. There are no reports regarding whether neonatal sevoflurane exposure-induced $\mathrm{BBB}$ disruption are dependent on exposure time and whether they are reversible. The present study was performed to examine the length of time required for neonatal sevoflurane exposure to cause BBB disruption in mice. Furthermore, we investigated whether these changes were reversible.

The Animal Care and Use Committee of Tokyo Medical and Dental University approved the protocol in the current study (0170118A). C57BL/6NCr strain mice (male and female) at POD6 were used for the study. To reduce variability, the same number of pups from each litter were used for the same experiment.

Corresponding author: Maiko Satomoto, M.D., Ph.D.

Department of Anesthesiology, Toho University School of Medicine, 6-11-1, Omori-Nishi Ota-ku, Tokyo 1438541, Japan

Tel: +81-3-3762-4151, Fax: +81-3-3765-8022

Email: maiko.satomoto@med.toho-u.ac.jp

ORCID: https://orcid.org/0000-0003-2768-7482

${ }^{*}$ Past address: Department of Anesthesiology, Tokyo Medical and Dental University, Graduate School of Medical and Dental Sciences, Tokyo, Japan

Received: January 1, 2019.

Revised: February 4, 2019.

Accepted: February 11, 2019.

Korean J Anesthesiol 2019 August 72(4): 389-391

https://doi.org/10.4097/kja.d.19.00029
Sevoflurane anesthesia was performed as previously described [2,3]. Briefly, POD6 mice were placed in a humid and warm chamber immediately after removal from the maternal cage. Sevoflurane (2\%) was administered with $40 \%$ oxygen as the carrier gas for $6 \mathrm{~h}$. Total gas flow was $1 \mathrm{~L} / \mathrm{min}$.

To study the effects of BBB disruption, POD6 mice were randomly divided into control or sevoflurane groups and exposed to carrier gas or sevoflurane, respectively (total number of pups used $=18$ ). $\mathrm{BBB}$ disruption in the hippocampal CA1 region were examined using a transmission electron microscope (TEM) (H-7100; Hitachi, Japan) at 2, 4, and 6 h during sevoflurane exposure, and 24 and $48 \mathrm{~h}$ after sevoflurane exposure. Furthermore, we evaluated POD16 mice and 8-week-old mice that were exposed to carrier gas or $2 \%$ sevoflurane for $6 \mathrm{~h}$.

Brain tissue were perfused with freshly prepared $4 \%$ paraformaldehyde and $2.5 \%$ glutaraldehyde. After dehydration, the hippocampal CA1 region was embedded in Epon 812, cut with a microtome, stained with uranyl acetate and lead citrate, and examined using TEM. We observed perivascular spaces because in our previous investigation [3] sevoflurane disrupted the perivascular spaces. We picked 15 capillaries in the hippocampal CA1 region for each animal sampled at each time point. The number of capillaries with destroyed perivascular space was counted and presented as a percentage of the number of capillaries with destroyed ultrastructure (\%). Values are expressed as mean \pm SD.

In the control group, the ultrastructure of capillaries in the CA1 region of the hippocampus was continuous, and the perivascular spaces were normal (Figs. 1A and $1 \mathrm{~F}$ ). However, the ultrastructural integrity was locally collapsed after $2 \mathrm{~h}$ of sevoflurane exposure (Figs. $1 \mathrm{~B}$ and $1 \mathrm{G}$ ). The perivascular spaces gradually became enlarged and collapsed depending on the sevoflurane exposure time (Figs. 1B-1D, 1G and 1H). To clarify

(c) This is an open-access article distributed under the terms of the Creative Commons Attribution Non-Commercial License (http://creativecommons.org/ licenses/by-nc/4.0/), which permits unrestricted non-commercial use, distribution, and reproduction in any medium, provided the original work is properly cited. 

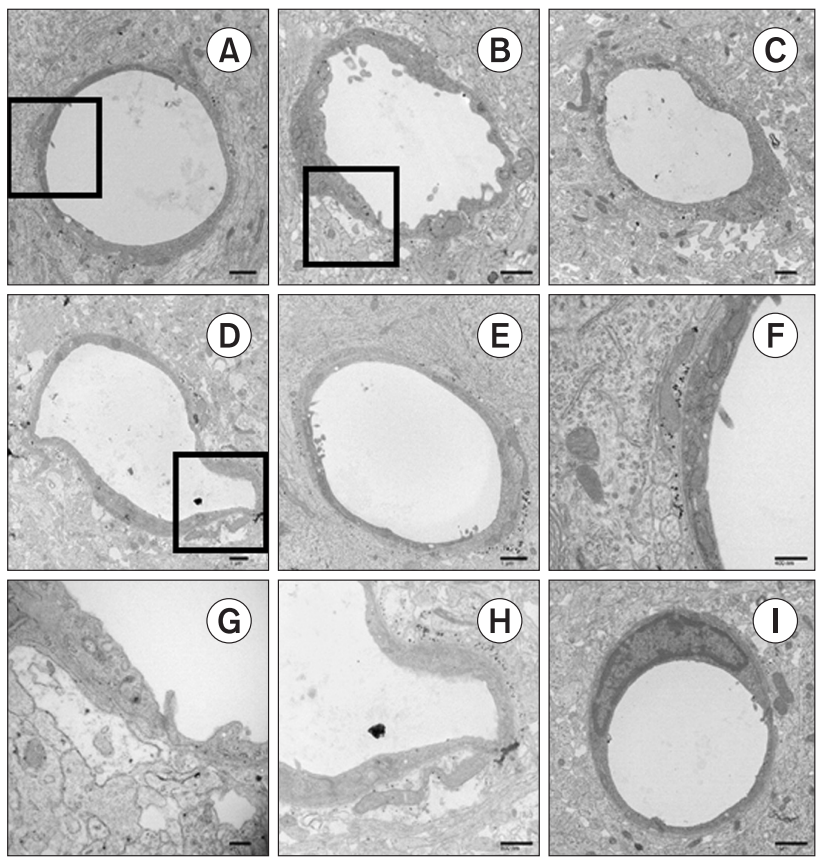

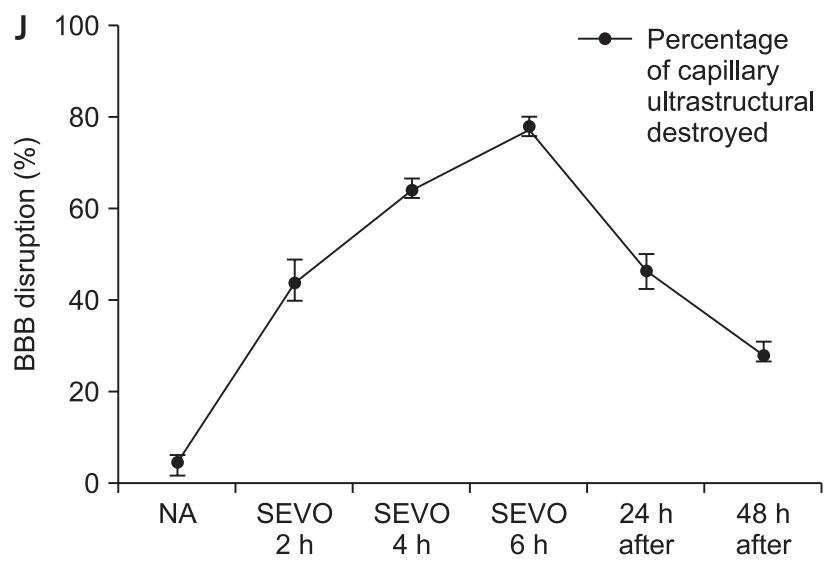

Fig. 1. The ultrastructure of the capillaries (A, F) in the control group, $(B, G)$ after sevoflurane exposure for $2 \mathrm{~h},(\mathrm{C})$ after sevoflurane exposure for $4 \mathrm{~h},(\mathrm{D}, \mathrm{H})$ after sevoflurane exposure for $6 \mathrm{~h},(\mathrm{E})$ after sevoflurane exposure for $24 \mathrm{~h}$, (I) after sevoflurane exposure for $48 \mathrm{~h}$, and (J) quantification of blood-brain barrier (BBB) disruption at each time point. The $\mathrm{X}$ axis shows sevoflurane exposure time and time after sevoflurane exposure for 6 $\mathrm{h}(24 \mathrm{~h}$ and $48 \mathrm{~h}$ after the end of anesthesia). In the control group, the capillaries were continuous and integrated, and the perivascular spaces were normal. However, the ultrastructural integrity of the BBB began to be disrupted after $2 \mathrm{~h}$ of sevoflurane exposure as perivascular spaces were enlarged. Sevoflurane exposure induced $\mathrm{BBB}$ opening in a duration-dependent manner. Additionally, the effect of sevoflurane exposure on the BBB opening was reversible as the enlarged perivascular spaces recovered gradually $24-48 \mathrm{~h}$ after sevoflurane exposure. The destroyed perivascular space was counted and presented as percentage of capillary ultrastructural destroyed (\%). Values are presented as mean \pm SD. $n=3$ in each group. (F, G, and H) show higher magnifications of the insets square in $(A, B$, and $D)$, respectively. Scale bars are shown on the right lower panels.

these changes, Fig. 1J shows the quantification of BBB ultrastructural abnormalities (\%). After $4 \mathrm{~h}$ of sevoflurane exposure, $64 \%$ of capillaries were destroyed and perivascular spaces were enlarged; $78 \%$ of capillaries were destroyed after 6 h of sevoflurane exposure.

Next, we examined the reversibility of the BBB disruption. Twenty-four hours after removal of sevoflurane, $47 \%$ of capillaries were destroyed (Figs. 1E and 1J), and this dropped to $29 \%$ after $48 \mathrm{~h}$ (Figs. 1I and 1J).

The most vulnerable period for neonatal mice is during the first 2 postnatal weeks, which is also known as the brain growth spurt (BGS) period in rodents [4]. Therefore, we investigated whether the BBB disruption is dependent on the age at which sevoflurane exposure occurs. At 16 days after birth, $6 \mathrm{~h}$ of sevoflurane exposure had no influence on the BBB disruption (data not shown). Furthermore, at 8 weeks old, there was no destruction of the perivascular space in the hippocampal CA1 region (data not shown).

We showed that $2 \%$ sevoflurane caused BBB disruption in the hippocampus of mice at POD6. These abnormalities occurred only during the period of neuronal proliferation in the brain. Furthermore, we showed that these BBB disruption following neonatal 2\% sevoflurane exposure were reversible.

The BBB was shown to open gradually with sevoflurane exposure time. Pups were returned to their dam after $6 \mathrm{~h}$ of anesthesia. At this time point, $78 \%$ of the BBB was disrupted, but this decreased to $47 \%$ after $24 \mathrm{~h}$ and to $29 \%$ after $48 \mathrm{~h}$. Therefore, we confirmed that the $\mathrm{BBB}$ recovered after $2 \%$ sevoflurane exposure in POD6 mice. Meanwhile, even with exposure to $2 \%$ sevoflurane for $6 \mathrm{~h}$, both POD16 and 8 week old mice showed no changes in the BBB. The neurotoxic effects appeared to occur during the period of brain development corresponding to synaptogenesis. This period corresponds to the BGS and is generally thought to occur between the third trimester of pregnancy and 3 years of age in humans, corresponding to the first 2 weeks postnatally in rodents [4]. Our results may explain the vulnerability of the developing brain to sevoflurane exposure.

This anesthetic protocol is often used in clinical practice in infants; however, a leaky BBB may lead to adverse outcomes under clinical conditions. It should be noted that there are differences between species, duration of anesthesia, and concentration of sevoflurane. Effects of anesthetics on rodents during BGS have been studied by exposing them to the anesthetic for long periods (4-6 h) [1-3]. A shorter period of exposure (30 
min) to sevoflurane did not induce obvious long-term effects [5]. Moreover, morphological changes were observed using TEM in a manner dependent on the duration of anesthesia.

The results of the present study clearly showed that $2 \%$ sevoflurane exposure during the BGS caused BBB disruption in the hippocampus of mice at POD6. In addition, the BBB disruption after neonatal sevoflurane exposure in POD6 mice were reversible.

\section{Conflicts of Interest}

No potential conflict of interest relevant to this article was reported.

\section{Author Contributions}

Zhongliang Sun (Investigation)

Maiko Satomoto (Data curation; Funding acquisition; Writing original draft; Writing-review \& editing)

Yushi U Adachi (Writing-review \& editing)

Koshi Makita (Supervision)

\section{ORCID}

Zhongliang Sun, https://orcid.org/0000-0002-3657-6963

Maiko Satomoto, https://orcid.org/0000-0003-2768-7482

Yushi U Adachi, https://orcid.org/0000-0001-8899-5708

Koshi Makita, https://orcid.org/0000-0002-1309-8102

\section{References}

1. Satomoto M, Satoh Y, Terui K, Miyao H, Takishima K, Ito M, Imaki J. Neonatal exposure to sevoflurane induces abnormal social behaviors and deficits in fear conditioning in mice. Anesthesiology 2009; 110: 628-37.

2. Sun Z, Satomoto M, Adachi YU, Kinoshita H, Makita K. Inhibiting NADPH oxidase protects against long-term memory impairment induced by neonatal sevoflurane exposure in mice. Br J Anaesth 2016; 117: 80-6.

3. Satomoto M, Sun Z, Adachi YU, Makita K. Sugammadex-enhanced neuronal apoptosis following neonatal sevoflurane exposure in mice. Anesthesiol Res Pract 2016; 2016: 9682703.

4. Dobbing J, Sands J. Comparative aspects of the brain growth spurt. Early Hum Dev 1979; 3: 79-83.

5. Qiu L, Zhu C, Bodogan T, Gómez-Galán M, Zhang Y, Zhou K, et al. Acute and long-term effects of brief sevoflurane anesthesia during the early postnatal period in rats. Toxicol Sci 2016; 149: 121-33. 